\title{
Los recursos humanos en las bibliotecas públicas de México: una investigación exploratoria
}

\author{
Federico Hernández Pacheco * \\ Diana Quezada Escamilla **
}

Artículo recibido:

13 de enero de 2014.

Artículo aceptado: 14 de mayo de 2015.

\section{Resumen}

Ante los nuevos retos que presenta la sociedad de la información, las bibliotecas públicas mexicanas requieren la participación de recursos humanos de alta calidad, que permitan satisfacer las demandas de sus usuarios e impulsar diversas iniciativas para la mejora continua de los servicios. El personal bibliotecario es un factor esencial; por ello, el interés de este trabajo es explorar el panorama actual de los recursos humanos en algunas de las bibliotecas que integran la Red Nacional de Bibliotecas Públicas de México.

* InstitutodelnvestigacionesBibliotecológicasydelalnformacióndelaUNAM, México.federicohp@iibi.unam.mx

* PosgradoenBibliotecologíayEstudiosdelalnformacióndelaUNAM,México. agricefala@comunidad.unam.mx AgradecemoseltrabajodeentrevistaspresencialesdeArieldelaRosaAlvaradoy Nayeli Castillo Moreno del Colegio de Bibliotecología de la UNAM.

INVESTIGACIÓN BIBLIOTECOLÓGICA,Vol.30,Núm.68,enero/abril,2016,México,ISSN: 0187-358X. pp. 17-50 
Palabras clave: Red Nacional de Bibliotecas Públicas; Bibliotecas públicas; Recursos humanos; México.

\begin{abstract}
Human resources in Mexican public libraries: an exploratory inquiry

FedericoHernández-Pachecoand Diana Quezada-Escamilla
\end{abstract}

Facing the new challenges of information society, Mexican public libraries need highly qualified human resources to satisfy demands of users and promote initiatives for ongoing improvement of library services. Library human resources are an essential part of public libraries; therefore, this research examines the human resources in several libraries of the National Network of Public Libraries in Mexico.

Keywords: National Network of Public Libraries; Public Libraries; Human Resources; Mexico.

\title{
Introducción
}

as bibliotecas públicas ocupan un lugar estratégico en la construcción del tejido social de nuestro país, ya que son necesarias para el desarrollo cultural, lúdico y educativo, aspectos clave no sólo para facilitar el ingreso de las personas a la denominada sociedad de la información y del conocimiento, sino para reducir las desigualdades que prevalecen en la actualidad.

En este sentido, es importante recordar que la biblioteca pública es democrática por excelencia, al facilitar servicios de carácter informativo y formativo en un contexto de inclusión y diversidad, de libertad y de paz, así como en un marco propicio para la convivencia que, no obstante los avances de las tecnologías, estará presente por muchos años en la cotidianidad de las poblaciones.

Sin embargo, resulta indispensable que toda biblioteca pública cuente, entre otras cosas, con un local o infraestructura adecuada, con recursos de información vastos yacordes a las necesidades locales, con una variedad de servicios, así como con recursos financieros justos, pero ante todo, debe tener personas 
debidamente formadas y con actividades correctamente planificadas a fin de garantizar el uso y manejo eficiente de todos los medios en beneficio de la biblioteca y de la comunidad a la que ésta sirve.

En efecto, se sabe de antemano que las instalaciones, el presupuesto, el equipo, el mobiliario, etc., son recursos básicos para el correcto funcionamiento de diversas organizaciones tanto públicas como privadas, y que son elementos sin los cuales sería muy difícil o hasta imposible llevar a cabo las tareas que se efectúan a diario en entornos laborales donde se intercambian bienes y servicios, como es el caso de las bibliotecas públicas y las interacciones que se dan con sus usuarios.

No obstante, existe un recurso clave del cual depende la operación adecuada de los medios con los que dispone una organización, y son los recursos humanos, quienes a través de su talento, trabajo, creatividad y esfuerzo pueden conducir a la organización hacia el éxito o el fracaso. ${ }^{1}$

Cabe destacar que la eficacia de los servicios que prestan muchas bibliotecas públicas depende, en gran medida, de las personas que trabajan en éstas, pues son quienes representan un papel preponderante en la mejora continua y en la calidad de los servicios de una comunidad determinada.

Ahora bien, existen diversas problemáticas en torno a los recursos humanos. En el caso de México, según el dato obtenido del 11o. Seminario Hispano-mexicano de Investigación en Bibliotecología y Documentación, ${ }^{2}$ en la actualidad 16000 personas laboran en la Red Nacional de Bibliotecas. El gobierno federal, los estados, municipios y delegaciones del Distrito Federal tienen la responsabilidad, en su ámbito territorial, de seleccionar, contratar, pagar salarios, capacitar y evaluar a dicho personal. No obstante, prevalecen situaciones que exhortan a elaborar estudios sobre los recursos humanos en bibliotecas públicas. Por ejemplo, en los municipios el personal se rota o sustituye cada tres años como consecuencia de los cambios de gobierno municipales. Lo mismo ocurre en los estados y en la federación, donde la rotación de personal es evidente cada seis años; esto obliga no solamente a formar al personal de nuevo ingreso, sino a invertir nuevamente en recursos públicos para esta formación y para otras funciones. Por otra parte, la mayor parte del personal que labora en estas bibliotecas percibe un salario que oscila entre los \$ 2000 y \$ 3000 ; aunado a ello, el nivel de escolaridad en su mayoría es de educación secundaria y preparatoria, sin alguna especialidad en el área de bibliotecología. Algunos aspectos desconocidos hasta el momento en relación a los recursos humanos son la manera en cómo se evalúa al personal,

1 F. Hernández Pacheco, Gestión y desarrollo de recursos humanos en bibliotecas, p. 15.

2 Celebrado del 23 al 25 de abril de 2014 en Ciudad Universitaria, UNAM, México. 
en qué medida los salarios se encuentran relacionados con el desempeño, la valuación y el perfil de puestos de trabajo, así como la forma en que se realiza el proceso de selección de los recursos humanos.

Al respecto, las Directrices IFLA/Unesco para el desarrollo del servicio de bibliotecas públicas señalan claramente:

La administración del personal bibliotecario es por sí sola una importante tarea. Todo el personal deberá tener un conocimiento exhaustivo de la política del servicio de bibliotecas, tareas y responsabilidades bien definidas, condiciones de empleo y salarios adecuadamente reglamentados y competitivos con los de otros empleos similares. ${ }^{3}$

Asimismo, la Organización para la Cooperación y el Desarrollo Económicos (OCDE) publicó el martes 18 de marzo la edición 2014 de su reporte Panorama de la sociedad, donde destaca que México, país que pertenece a la OCDE desde 1994, tiene el menor ingreso disponible entre los países del bloque. De igual forma, el reporte advierte que la desigualdad del ingreso y las divisiones sociales pueden empeorar y volverse persistentes a menos que los gobiernos actúen rápidamente para impulsar el apoyo a los sectores más vulnerables de la sociedad. ${ }^{4}$

En todo este contexto, la Ley General de Bibliotecas en México señala en su artículo 7o., apartado X, que corresponde a la Secretaría de Educación Pública "proporcionar entrenamiento y capacitación al personal adscrito a las bibliotecas públicas de la Red", función que viene realizando el Conaculta desde su creación a través de su Dirección General de Bibliotecas.

Además, en su artículo 8o. establece que corresponde a los gobiernos de los estados:

VI. Designar al coordinador de la Red Estatal quien fungirá como enlace con la Coordinación de la Red nacional de Bibliotecas Públicas.

VII. Nombrar, adscribir y remunerar al personal destinado a la operación de sus bibliotecas públicas.

Sin embargo, es de notar que estos enunciados no aclaran lo referente al desarrollo integral de los recursos humanos, que va más allá de la adscripción, la designación, el entrenamiento o capacitación y la remuneración. Por ello, es importante dotar de una formación continua al personal, en el marco de otras funciones básicas de los recursos humanos como son la planeación estratégica de los puestos, el reclutamiento y la selección basados en aspec-

3 Directrices IFLA-Unesco para el desarrollo del servicio de bibliotecas públicas.

4 Roberto González Amador, "En México 4 de cada diez personas no tienen para comprar alimentos". 
tos como la escolaridad o la preparación académica, considerando además las habilidades o las competencias para el trabajo. Asimismo, es importante tener en cuenta el análisis, la descripción y la valuación de puestos; los planes de compensación o retribución justos y equitativos, la evaluación del rendimiento y del potencial, la promoción del personal o desarrollo de carreras, la comunicación efectiva y el clima laboral, la administración del talento, la ergonomía y la prevención de riesgos laborales, así como las relaciones humanas y laborales, entre otros aspectos.

Bajo estas premisas se consideró conveniente realizar un estudio en algunas bibliotecas públicas pertenecientes a la RNBP para explorar la problemática y brindar una serie de propuestas para la mejora de los recursos humanos $y$, por ende, de la calidad en todos los procesos y servicios de las bibliotecas públicas del país.

De esta manera, en este estudio se lograron respuestas de forma general y en primera instancia a las siguientes preguntas: ¿cuáles son las características del personal que labora en las bibliotecas públicas seleccionadas? ¿Cuál es la preparación, condiciones laborales, habilidades y actividades de los recursos humanos en las bibliotecas públicas seleccionadas? ¿Cuáles son las principales problemáticas que se presentan en materia de recursos humanos en bibliotecas públicas? Para ello, se establecieron los siguientes objetivos:

- Conocer las características generales del personal de algunas bibliotecas públicas de México.

- Detectar problemas que aquejan a los recursos humanos en las bibliotecas públicas de México.

- Proporcionar sugerencias, para abordar en investigaciones posteriores, vinculadas el desarrollo de los recursos humanos en las bibliotecas públicas mexicanas.

En estas condiciones, la presente investigación exploratoria y descriptiva ha servido para obtener información que ayudará a profundizar en los diversos aspectos y funciones de los recursos humanos en las bibliotecas públicas mexicanas. Su continuación en otros estudios correlacionales y explicativos servirá para la elaboración de proyectos para la mejora de las condiciones del personal y contribuirá a llenar los huecos de carácter teórico y práctico en esta materia.

Lo que aquí se reporta da a conocer los primeros resultados de las entrevistas y el levantamiento de información que se trabajó en algunas bibliotecas de la RNBP con el fin de tener elementos que sirvan para establecer a futuro un modelo de desarrollo de recursos humanos que se pueda replicar en el país. 
Los beneficiados de este estudio serán principalmente las personas que trabajan en las bibliotecas públicas mexicanas, así como aquellos directivos o personas en quienes recae la toma de decisiones y que opten por incorporar este esquema para detectary brindar soluciones a las problemáticas actuales en la materia que aquí atañe. Con ello, se reconoce la importancia que tienen los recursos humanos, su impacto social y la manera en que se puede favorecer la difusión y consumo de la cultura con el apoyo de las personas que atienden las bibliotecas.

\section{Definiciones y aspectos generales sobre biblioteca pública y recursos humanos}

\section{Biblioteca pública}

Según el Manifiesto de la IFLA/Unesco sobre la biblioteca pública de 1994, la biblioteca pública es:

un centro local de información que facilita a sus usuarios todas las clases de conocimiento e información.

Los servicios de la biblioteca pública se prestan sobre la base de igualdad de acceso para todas las personas, sin tener en cuenta su edad, raza, sexo, religión, nacionalidad, idioma o condición social. Deben ofrecerse servicios y materiales especiales para aquellos usuarios que por una u otra razón no pueden hacer uso de los servicios y materiales ordinarios, por ejemplo, minorías lingüísticas, personas con discapacidades o personas en hospitales o en prisión.

Todos los grupos de edad han de encontrar material adecuado a sus necesidades. Las colecciones y los servicios han de incluir todo tipo de soportes adecuados, tanto en modernas tecnologías como en materiales tradicionales. Son fundamentales su alta calidad y adecuación a las necesidades y condiciones locales. Los materiales deben reflejar las tendencias actuales y la evolución de la sociedad, así como la memoria del esfuerzo y la imaginación de la humanidad. Ni los fondos ni los servicios han de estar sujetos a forma alguna de censura ideológica, política o religiosa, ni a presiones comerciales. ${ }^{5}$

La definición anterior, aun cuando data de hace 20 años, sigue vigente al tener en cuenta la igualdad dentro de la biblioteca pública como un valor fundamentado en el trato idéntico entre todas las personas, así como en la inclusión, un concepto que tiene como eje el logro de oportunidades y la 
distribución de recursos de manera equitativa para disfrutar de calidad de vida y bienestar. Por otra parte, también resalta las características de las colecciones o materiales bibliográficos en la biblioteca pública, en función de la diversidad de intereses y necesidades de la comunidad misma a la que ésta pertenece.

El documento Directrices IFLA/Unesco para el desarrollo del servicio de bibliotecas públicas apunta lo siguiente:

es una organización establecida, respaldada y financiada por la comunidad, ya sea por conducto de una autoridad u órgano local, regional o nacional o mediante cualquier otra forma de organización colectiva. Brinda acceso al conocimiento, a la información y al trabajo intelectual a través de una serie de recursos y servicios y está a disposición de todos los miembros de la comunidad en igualdad de condiciones sin distinción de raza, nacionalidad, edad, sexo, religión, idioma, discapacidad, condición económica y laboral y nivel de escolaridad. ${ }^{6}$.

Esta definición nos brinda un elemento más: el respaldo económico que, en la mayoría de los casos, se realiza con el erario público, lo que concreta a esta entidad como una institución para todos.

Por otra parte, la biblioteca pública adquiere un valor esencial al incluir la presencia de una gran diversidad de público usuario, hecho que la IFLA/ Unesco señala en las últimas líneas de su definición, con lo que se le confiere un estatus igualitario. En nuestro país, tenemos que la Ley General de Bibliotecas define a la biblioteca pública en su artículo 20. como:

todo establecimiento que contenga un acervo impreso o digital de carácter general superior a quinientos títulos, catalogados y clasificados, y que se encuentre destinado a atender en forma gratuita a toda persona que solicite la consulta o préstamo del acervo en los términos de las normas administrativas aplicables.

La biblioteca pública tendrá como finalidad ofrecer en forma democrática el acceso a los servicios de consulta de libros, impresos y digitales, y otros servicios culturales complementarios, como orientación e información, que permitan a la población adquirir, transmitir, acrecentar y conservar en forma libre el conocimiento en todas las ramas del saber.

En el enunciado anterior destacan los conceptos de gratuidad en la prestación de los servicios, de democracia al dar acceso a todas las personas y formas de expresión, y de libertad al hacer uso de los materiales con independencia, fomentando el trabajo intelectual y colaborativo; no obstante, la definición es limitada tomando en cuenta que data de 1988. 
Por su parte, el artículo 2o. de la Ley de Bibliotecas del Distrito Federal ofrece las definiciones siguientes:

V. Biblioteca. El espacio físico que cuenta con una estructura organizativa, un acervo bibliográfico, hemerográfico y documental en cualquier formato, tecnologías de la información y comunicación, cuya misión es contribuir al desarrollo de las personas y su calidad de vida por medio de la difusión del pensamiento, el acceso a la lectura, la información, la investigación, las expresiones culturales en igualdad de oportunidades a toda persona que la visite.

VI. Biblioteca pública. Cualquier biblioteca dependiente de la administración pública del Distrito Federal que preste servicios al público en general.

En realidad la primera definición se acerca más a la misión y objetivos de la biblioteca pública, mientras que la concerniente específicamente a la biblioteca pública carece de un tratamiento mayor acerca de las características de una biblioteca de este tipo: la gratuidad de los servicios, la inclusión de los miembros de la comunidad y la amplia temática de sus colecciones, entre otros aspectos.

Otras cualidades que posee la biblioteca pública son las siguientes:

- Almacena, organiza y suministra toda información contenida en cualquier tipo de soporte documental proveniente de fuentes autorizadasy fiables.

- Dispone de información local y global que es útil para la comunidad a la que sirve. Esto plantea que debe afrontar retos constantes en materia de acceso, cobertura, amplitud y profundidad.

- Brinda servicios culturales y artísticos que permiten a la población adquirir, acrecentar y transmitir conocimientos para su desarrollo personal.

- Cumple una función educativa que la posiciona como una institución de gran calibre, pues a través de sus servicios ofrece la coyuntura para transitar a una sociedad más informada, participativa, justa y libre.

Tomando como referente las diferentes definiciones que han sido expuestas, se puede concluir que la biblioteca pública es un espacio social que contiene colecciones de materiales impresos o en formato digital sobre temas de interés general, cuya función primordial es la de servir a una localidad por medio de diferentes servicios de índole informativo y cultural, los cuales deben prestarse de manera gratuita y libre bajo ciertos lineamientos. Dichos servicios se hacen posibles a través de diversas estrategias destinadas a los usuarios y realizadas por el personal con que cuenta la biblioteca, el cual de- 
be estar debidamente preparado, motivado y contar con las condiciones laborales adecuadas para el desempeño de sus actividades.

\section{Recursos humanos}

A los recursos humanos se les identifica como el conjunto de personas que laboran y forman parte de una organización pública o privada, en donde llevan a cabo diversas tareas que les son asignadas de acuerdo a sus capacidades, sus talentos y conforme las necesidades que se presenten a diario. Dessler y Varela señalan que

la administración de los recursos humanos se refiere a las prácticas y políticas necesarias para manejar los asuntos que tienen que ver con las relaciones humanas del trabajo administrativo; en específico, se trata de reclutar, capacitar, evaluar, remunerar y ofrecer un ambiente seguro y equitativo para los empleados. ${ }^{7}$

\section{Por su parte Chiavenato afirma que}

los recursos humanos están distribuidos en el nivel institucional de la organización (dirección), en el nivel intermedio (gerencia y asesoría) y en el nivel operacional (técnicos, auxiliares y operarios). El recurso humano -continua- es el único recurso vivo y dinámico de la organización y decide el manejo de los demás, que son físicos y materiales. Además constituye un tipo de recurso que posee una vocación encaminada hacia el crecimiento y desarrollo. ${ }^{8}$

Ambos conceptos invitan a reflexionar acerca de la importancia de las personas como el elemento clave de las organizaciones en un mundo cambiante y globalizado. Igualmente, el manejo u operación de personas requiere de una eficiente organización y de diversas funciones o prácticas totalmente necesarias para el logro de los objetivos.

En este contexto, el Manifiesto de la IFLA/Unesco sobre la biblioteca pública de 1994 señala que:

La biblioteca pública ha de organizarse eficazmente y mantener normas profesionales de funcionamiento. El bibliotecario es un intermediario activo entre los usuarios y los recursos. Es indispensable su formación profesional y permanente para que pueda ofrecer servicios adecuados. ${ }^{9}$

7 Gary Dessler y Ricardo A. Varela Juárez, Administración de recursos humanos: enfoque latinoamericano, p. 2.

8 Idalberto Chiavenato, Administración de recursos humanos, p. 128.

9 Federación Internacional de Asociaciones de Bibliotecarios y Bibliotecas, op. cit. 

Asimismo, las Directrices IFLA/Unesco para el desarrollo del servicio de bi- bliotecas públicas reconocen lo siguiente:

El personal es un recurso de vital importancia en el funcionamiento de una biblioteca [...] Para prestar servicios óptimos a la comunidad es necesario mantener un personal capacitado y muy motivado a fin de utilizar eficazmente los recursos de la biblioteca en respuesta a las demandas de la comunidad [...]

Todo el personal deberá tener un conocimiento exhaustivo de la política del servicio de bibliotecas, tareas y responsabilidades bien definidas, condiciones de empleo y salarios adecuadamente reglamentados y competitivos con los de otros empleos similares. [...]

El personal de una biblioteca pública deberá contar con una serie de aptitudes y cualidades, entre ellas sociabilidad, conciencia social, capacidad de trabajar en equipo y dominio y competencia con respecto a las prácticas y procedimientos de la institución. ${ }^{10}$

Tanto el Manifiesto como las Directrices reconocen la relevancia de las personas en el manejo óptimo de la biblioteca pública, ya que además "son proveedoras de conocimientos, habilidades, competencias $y$, sobre todo, de la aportación más importante para las organizaciones: la inteligencia [...] las personas constituyen el capital intelectual de la organización".11 Un aspecto que indudablemente supera cualquier otro recurso con el que se cuente.

Las personas, en definitiva, serán el activo más importante de la biblioteca pública, por lo que deberán tener una formación continua con una visión y trabajo anticipado a las necesidades de los servicios, pero también deberán contar con los elementos suficientes que brindan un adecuado desarrollo y una eficiente administración de los recursos humanos.

\section{Método}

El presente trabajo de investigación es de tipo exploratorio-descriptivo, ya que pretende efectuar un primer acercamiento a la situación actual de los recursos humanos en las bibliotecas públicas pertenecientes a la Red Nacional de Bibliotecas Públicas de México.

El curso metodológico seguido en este estudio se basa en la utilización tanto de métodos cuantitativos como cualitativos. De esta manera, la técnica cuantitativa utilizada consistió en la aplicación de una encuesta en línea a una 
muestra determinada de bibliotecas a las que se hizo llegar el instrumento de recolección de datos; por otra parte, las técnicas cualitativas que se emplearon fueron la técnica de observación directa, de observación participante y de entrevista en los entornos en los que se desempeña el personal bibliotecario.

\section{Unidad de análisis}

El objeto de estudio de esta investigación comprende la población de recursos humanos que labora en las 7388 bibliotecas públicas pertenecientes a la Red Nacional de Bibliotecas Públicas de México. Dicha población se encuentra diversificada y, ante la falta de un registro oficial preciso y actualizado del personal que labora en dichos recintos, la investigación ha tomado en cuenta, en su conjunto, los diversos tipos de recursos humanos: ya sea el personal asalariado perteneciente al sindicato y de confianza, el personal que labora por voluntariado, así como el personal de servicio social y prácticas profesionales, entre otros.

\section{Muestra}

Dada la diversidad de la población y el tamaño de la misma, ha sido imprescindible seleccionar una muestra representativa del conjunto. A continuación, se explican el método, tipo y tamaño de la misma.

\section{Método de muestreo}

Con el propósito de alcanzar los objetivos trazados y mantener la representatividad de la población, se planteó que los elementos de la población que hubieran de integrar la muestra serían seleccionados a través del muestreo probabilístico con reemplazo, en el cual todas las bibliotecas de la Red tendrían la misma probabilidad de ser seleccionadas para formar parte de la muestra.

\section{Tipo de muestreo}

Para realizar la extracción probabilística, el tipo de muestreo a utilizar fue el aleatorio, en el cual se obtuvo la posibilidad de recibir respuestas de cualquier biblioteca pública en la República mexicana, resultando una muestra heterogénea en cuanto a la presencia de bibliotecas delegacionales, bibliotecas públicas centrales estatales, bibliotecas regionales y bibliotecas municipales. 
Tamaño de la muestra

La determinación del tamaño de la muestra se realizó a partir de la fórmula:

$$
n=\frac{N p q Z^{2}}{E^{2}(N-1)+p q Z^{2}}
$$

donde:

$\mathrm{N}$ (Tamaño de la población finita) $=7388$ bibliotecas públicas

$Z$ (Nivel de confianza) $=90 \%$

$E$ (Margen de error) $=5 \%$

$\mathrm{p}($ Variabilidad $)=40 \%$

$\mathrm{q}($ Complemento de la variabilidad $)=60 \%$

Lo anterior da como resultado un total de $n=249$ bibliotecas públicas.

\section{Instrumento de recolección de datos}

La técnica cuantitativa empleada en esta investigación consistió en la encuesta. Se aplicó un instrumento de recolección de datos constituido por un cuestionario en línea que puede ser consultado a través del enlace http://es.sur veymonkey.com/s/CJ5F5KC

Dicho cuestionario aborda la situación en torno a los recursos humanos, a partir de las siguientes variables de investigación:

1. Características demográficas.

2. Escolaridad.

3. Situación laboral.

4. Habilidades para el trabajo.

5. Funciones que realizan.

El instrumento se encuentra estructurado en diez reactivos (véase Anexo): el primero (1) lo constituyen cuatro campos abiertos enfocados al conocimiento de la biblioteca pública en la que labora quien responde la encuesta, así como el cargo o puesto de trabajo que desempeña y la ubicación de su biblioteca (municipio o delegación y entidad federativa).

Las siguientes dos preguntas o reactivos (2 y 3 ) se enfocan a responder a la primera variable de investigación: las características demográficas del personal, es decir, edad y género, respectivamente. 
La segunda variable, que trata sobre la escolaridad del personal, se estructuró en el reactivo 4, compuesto por ocho diferentes opciones que van en un rango desde personas sin estudios hasta las que tienen nivel de doctorado.

La tercera variable, concerniente a la situación laboral, se subdivide en cuatro diferentes rubros: horario laboral, salario que perciben los bibliotecarios, antigüedad que poseen como trabajadores de la biblioteca y la capacitación que reciben, aspectos que son tratados en las preguntas 5, 6, 7 y 8 .

La cuarta variable, habilidades para el trabajo, se trata en la pregunta 9, donde se brinda un listado de seis diferentes habilidades básicas que debe poseer el personal bibliotecario para el desempeño de su trabajo. La medición se efectúa a partir de una escala en donde el encuestado debe responder si se considera bueno, regular o malo en cada una de las destrezas propuestas.

Por último, el reactivo 10 se adscribe a la valoración de la quinta variable en cuanto a las funciones que realiza el personal. Para ello se estudiaron las actividades que deberían estar presentes en el desempeño del personal de toda biblioteca pública, por lo que se enumeró un listado con una serie de tareas de índole administrativa, técnica, auxiliar, etc., en las que el encuestado debía seleccionar aquellas que realiza con mayor frecuencia.

\section{Aplicación del instrumento}

Se optó por aplicar la encuesta a través de medios electrónicos. De esta manera, el instrumento de recolección de datos se diseñó con el software Survey Monkey. La estrategia para su aplicación consistió en hacer llegar el instrumento de recolección de datos a cada una de las 31 bibliotecas centrales estatales del país, por lo que fue necesario consultar el Directorio de Bibliotecas Centrales Estatales de la RNBP, disponible en http://www.rednacionaldebi bliotecas.gob.mx/mapa_biblioteca_estalRed.php

A partir de la información obtenida en dicho directorio, se envió un correo electrónico a cada una de las bibliotecas centrales estatales, exponiendo los objetivos del estudio y solicitando su colaboración para responder el cuestionario y, a su vez, hacerlo llegar a las bibliotecas municipales y regionales de su respectivo estado.

El enlace al cuestionario en línea estuvo disponible a partir del 7 de julio de 2013 y las respuestas comenzaron a recibirse a partir del día 22 del mismo mes, concluyendo la recopilación el 14 de octubre de 2013. 


\section{Limites del estudio}

El desarrollo de la investigación se vio afectado por tres principales factores:

a) Límites geográficos. La extensión territorial de la República mexicana hace difícil la realización de un estudio de campo que permita conocer la situación de los recursos humanos en cada una de las bibliotecas públicas del país de manera presencial y con un límite de tiempo preestablecido. Por ello se ha tenido que recurrir a la aplicación de instrumentos de recolección de datos de manera electrónica, con lo cual los resultados pudieron sesgarse.

b) Deficiencias de comunicación y participación. Una de las mayores problemáticas a las que se enfrentó esta investigación fue la falta de cooperación en los funcionarios de las bibliotecas centrales estatales. Este hecho pudo constatarse debido a la escasa recepción de respuestas en el cuestionario electrónico; mientras algunos estados se mostraron bastante participativos, otros jamás proporcionaron respuesta alguna. Lo anterior repercutió de manera notable en el análisis de resultados, ya que al haber un mayor número de respuestas en algunas entidades que en otras, la tendencia de los datos estuvo influenciada.

c) Peligro en la integridad de la personas. Uno de los factores limitantes que incidieron en la investigación cualitativa para el levantamiento de los datos in situ fue la situación delictiva y violenta que prevalece en diversas regiones del país, quedando fuera del proceso aquellas zonas peligrosas, de alto índice delictivo y lugares inaccesibles.

Por las limitantes expuestas, la presente investigación se establece, por el momento, como un primer estudio exploratorio-descriptivo en torno a la situación de los recursos humanos que laboran en las bibliotecas públicas del país. Los resultados obtenidos permitirán dar una orientación para futuras investigaciones a este respecto $y$, a su vez, fungirán como un acercamiento al tema de investigación aquí tratado.

\section{Corpus de bibliotecas estudiadas}

Del total de cuestionarios enviados a las 31 bibliotecas centrales estatales pertenecientes a la RNBP, sólo se recibió respuesta de Campeche, Durango y Jalisco.

Para complementar los resultados obtenidos mediante la encuesta electrónica, se consideró imprescindible la visita presencial a algunas bibliotecas públicas que por su cercanía y representatividad serían susceptibles de 
aportar datos que se pudieran incluir en la investigación. Para este efecto se hicieron visitas, en el Distrito Federal, a la Biblioteca Pública Francisco Zarco de la delegación Benito Juárez por el impacto social y cultural que se sabe ha tenido en la comunidad, y a la Biblioteca Juventino Rosas de la delegación Magdalena Contreras por los estándares ergonómicos y de buenas prácticas que posee. En el Estado de México se visitó la Biblioteca Beatriz Hampson Esquivel, del municipio de Ixtapaluca.

El instrumento de recolección de datos mencionado con anterioridad se aplicó al personal que labora en las bibliotecas del Distrito Federal y en la biblioteca del Estado de México. En total, la encuesta se aplicó a un corpus de 35 trabajadores pertenecientes a 10 bibliotecas públicas de cinco diferentes estados del país.

En la Tabla 1 se presenta la relación de bibliotecas participantes.

Tabla 1. Corpus de bibliotecas estudiadas

\begin{tabular}{|c|c|c|c|}
\hline Entidad federativa & Municipio/delegación & Biblioteca pública & Tipo \\
\hline Campeche & $\begin{array}{c}\text { San Francisco de } \\
\text { Campeche }\end{array}$ & FranciscoSosaEscalante & Central Estatal \\
\hline Distrito Federal & Benito Juárez & Francisco Zarco & Central Delegacional \\
\hline Distrito Federal & Magdalena Contreras & Juventino Rosas & Central Delegacional \\
\hline Durango & Vicente Guerrero & Octavio Paz & Municipal \\
\hline Durango & Vicente Guerrero & Tohue & Municipal \\
\hline Durango & Vicente Guerrero & $\begin{array}{c}\text { José Guadalupe } \\
\text { Rodríguez }\end{array}$ & Municipal \\
\hline Durango & Vicente Guerrero & José Ángel Ceniceros & Municipal \\
\hline Durango & Nombre de Dios & AlbertoTerronesBenítez & Regional \\
\hline Estado de México & Ixtapaluca & Beatriz Hampson & Municipal \\
\hline Jalisco & Guadalajara & Profr.RamónGarcíaRuiz & Central Estatal \\
\hline
\end{tabular}

\section{Resultados}

\section{Primer variable. Caracteristicas demográficas}

Se observa que la cuarta parte de los trabajadores encuestados tiene entre 30 y 39 años, mientras que poco más de la tercera posee entre 40 y 49 años; estos dos grupos por edad conforman el $71.43 \%$, según puede constatarse en la Figura 1 (p. sig.). Con relación a los datos obtenidos, puede afirmarse que los adultos mayores que laboran en bibliotecas públicas son una minoría, mientras que los jóvenes de entre 21 y 29 años constituyen alrededor del $17 \%$. Gracias a las visitas realizadas a las bibliotecas delegacionales del Distrito Federal y a las técnicas cualitativas empleadas, se puede ver que la labor 
desempeñada por los jóvenes en las bibliotecas públicas responde al servicio social que éstos realizan en dichas instituciones, además, son los trabajadores jóvenes quienes efectúan tareas principalmente en los Módulos de Servicios Digitales, lo cual establece una relación directa de esta variable con la formación así como con las funciones que realizan.

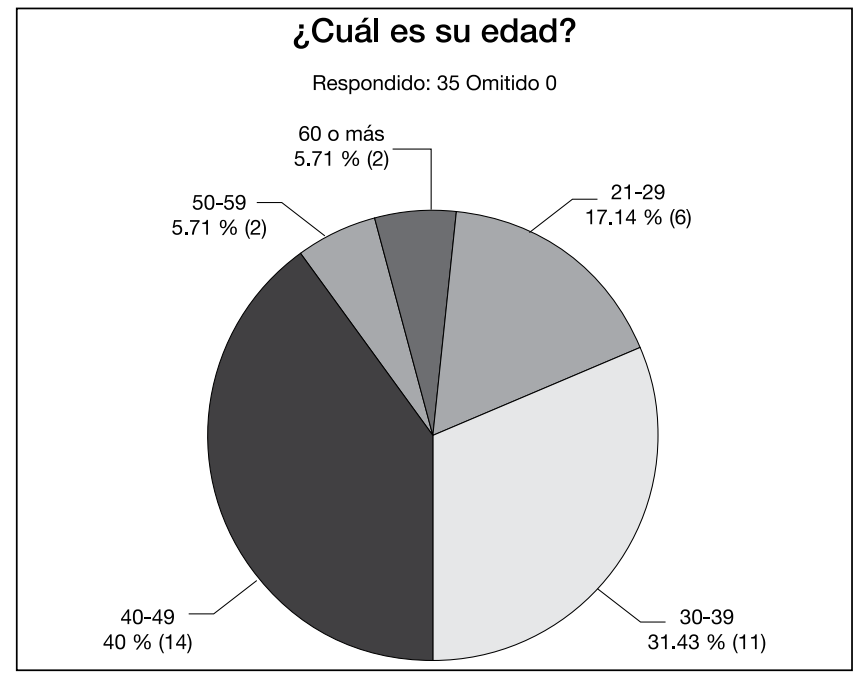

Figura 1. Gráfico de edad

Asimismo, es contundente que casi el $90 \%$ de los recursos humanos que laboran en las bibliotecas públicas del país son del género femenino (Figura 2, p. sig.). En las bibliotecas del Estado de México y el Distrito Federal puede observarse una mayor diversidad, pues trabajan en igual proporción tanto hombres como mujeres; sin embargo, en el caso del interior de la República, la totalidad de las respuestas que hasta ahora han sido obtenidas de los estados de Jalisco, Campeche y Durango corresponde a mujeres.

\section{Segunda variable. Escolaridad}

La formación es considerada un elemento de vital importancia para integrar a las personas a sus puestos de trabajo, así como para que puedan desempeñar sus actividades de una manera más productiva; es además, una estrategia organizacional ideal para desarrollar competencias, fomentar la creatividad de las personas e incrementar la innovación de las mismas. ${ }^{12}$ 


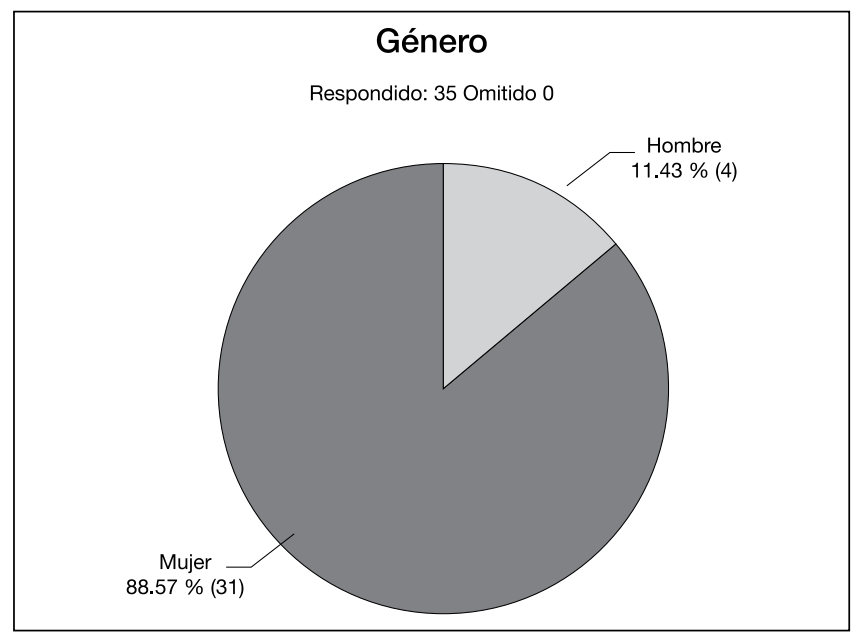

Figura 2. Gráfico de género

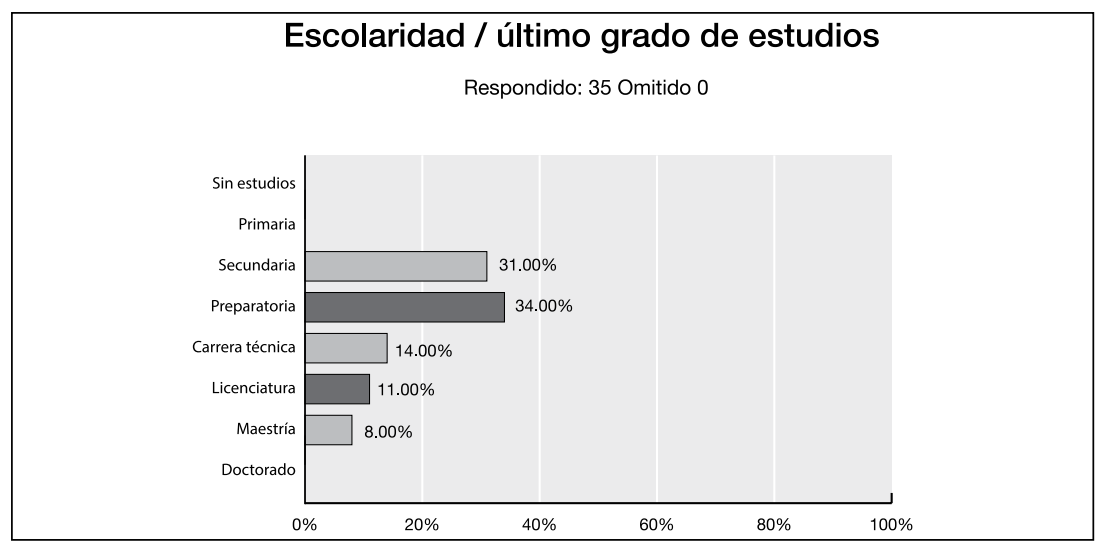

Figura 3. Gráfico de escolaridad

Por lo anterior, resulta de suma importancia que el personal cuente con una formación general y especializada, a fin de dar sustento a los procesos que tienen lugar en las bibliotecas públicas, no sólo en cuestión del desarrollo de colecciones o nuevas tecnologías, sino también en materia de servicios.

En el caso de las bibliotecas encuestadas, la escolaridad máxima de los bibliotecarios públicos oscila mayoritariamente en la educación media básica y media superior, esto es, la educación secundaria y preparatoria, con el 31 \% y el $34 \%$, respectivamente (Figura 3 ). 
Las Directrices IFLA/Unesco para el desarrollo del servicio de bibliotecas públicas sostienen que "los bibliotecarios deben haber realizado estudios universitarios o de posgrado en bibliotecología y ciencias de la información en una escuela de bibliotecología" ${ }^{\prime 13}$ sin embargo, como puede observarseen la Figura 3, los bibliotecarios que cuentan con estudios de licenciatura (11\%) constituyen una minoría, mientras que son aún menos los que poseen estudios de maestría (8\%).

Es de señalar que la mayoría de las personas no cuentan con una formación profesional en bibliotecología o ciencias de la información; por el contrario, los profesionales que laboran en bibliotecas públicas suelen poseer una formación en disciplinas distintas, entre ellas Pedagogía, Economía, Nutrición, Derecho, Administración y Contaduría, como se desprende de la encuesta y de las visitas presenciales.

\section{Tercer variable. Situación laboral}

Para efectos del estudio en cuanto a la situación laboral de los bibliotecarios, se han contemplado los siguientes aspectos:

1. Antigüedad laboral

2. Horario

3. Capacitación para el trabajo

4. Salario

\section{Antigüedad laboral}

Se entiende por antigüedad laboral el tiempo que el personal lleva trabajando en una institución, en este caso, la biblioteca pública; esta variable involucra la experiencia del bibliotecario en el puesto, ya que se considera que mientras mayor sea la antigüedad que tenga en su trabajo, mejor sabrá desempeñarse en éste.

Como puede observarse en la Figura 4 (p. sig.), el $48.57 \%$ de los recursos humanos tiene cinco años o menos trabajando en la biblioteca pública, es decir, que actualmente casi la mitad del personal que trabaja en las bibliotecas públicas es de nuevo ingreso, ya sea porque lo han transferido a esta área recientemente o bien por el cambio de administración local (municipal, estatal o delegacional). 


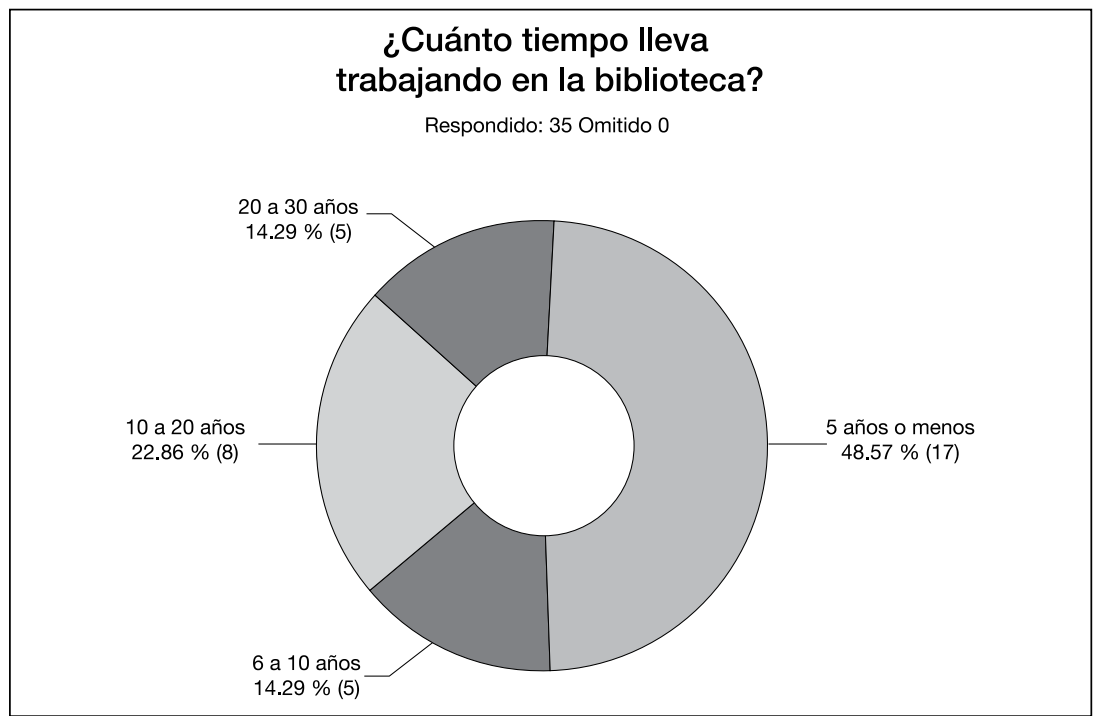

Figura 4. Gráfico de antigüedad

Por otra parte, el $22.86 \%$ afirma tener una antigüedad de 10 a 20 años de trabajo en la biblioteca, seguido del $14.29 \%$ de quienes poseen entre 6 y 10 años de trabajo. Al revisar y cotejar los resultados de esta variable, ha podido constatarse que quienes seleccionaron estas dos categorías de antigüedad (6 a 10 y 10 a 20) fueron los encargados y coordinadores de las bibliotecas estudiadas. Por lo tanto, se tiene que mientras mayor sea la antigüedad laboral que posean los bibliotecarios, mayores posibilidades tienen de ser encargados o coordinadores de la biblioteca.

\section{Horario laboral}

El Manifiesto de la Unesco sobre la biblioteca pública afirma que para el buen funcionamiento de esta institución es necesario ofertar una amplia gama de servicios dentro de un espacio adecuado, contemplando un horario suficiente y apropiado. ${ }^{14}$

Los datos obtenidos de este estudio muestran que casi la totalidad del personal trabaja en la biblioteca pública de lunes a viernes $(97 \%)$, mientras que sólo el $3 \%$ de los bibliotecarios señala trabajar en fines de semana (Figura 5, p. sig.). Lo anterior se contrapone con lo afirmado por las Directrices IFLA/Unesco para el desarrollo del servicio de bibliotecas públicas: "para pro-

14 Federación Internacional de Asociaciones de Bibliotecarios y Bibliotecas, op. cit., p. 3. 
porcionar el mejor acceso posible a sus servicios, la biblioteca ha de ofrecer el horario más práctico a quienes viven, trabajan o estudian en la comunidad". ${ }^{15}$

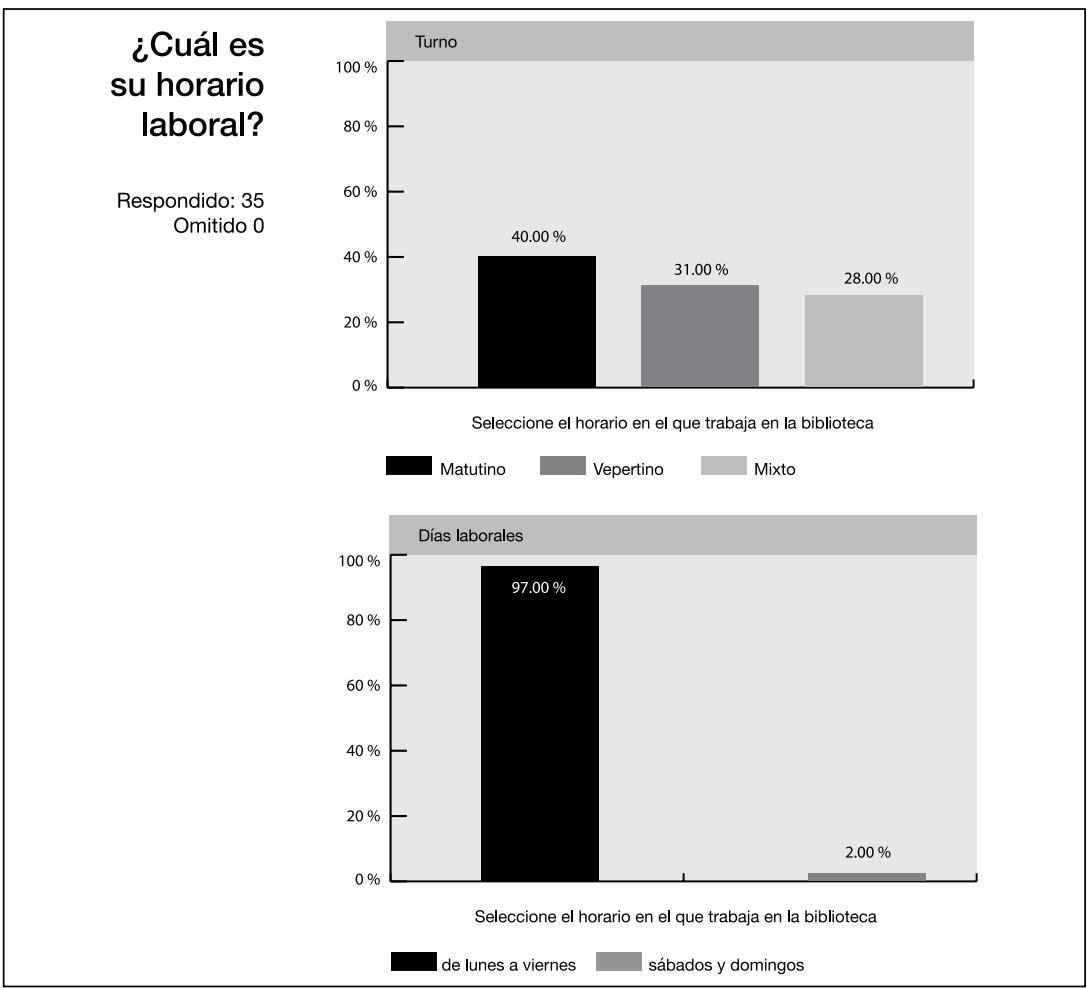

Figura 5. Gráfico de horario laboral

De esta manera se tiene que con un horario de apertura que sólo cubre los días laborales o escolares (de lunes a viernes) se está excluyendo a una gran parte de la población susceptible de utilizar los servicios bibliotecarios, ya que la biblioteca pública no es sólo un centro de información y complemento educativo, sino también una fuente de recreación y ocupación del tiempo libre, por lo que debería contemplarse su apertura durante los fines de semana. ${ }^{16}$

En cuanto al turno laboral, el $40 \%$ del personal encuestado indica trabajar en el turno matutino, mientras que el $31 \%$ en el turno vespertino. Los

15 Gill, op. cit., p. 40.

16 Esta propuesta conlleva una serie de esfuerzos: ampliar la plantilla de personal para fin de semana y, por ende, ampliar el presupuesto destinado a las bibliotecas públicas; sin embargo, ello parte de una reformulación de las políticas bibliotecarias urgentes en nuestro país. 
coordinadores de las bibliotecas centrales delegacionales del Distrito Federal refieren que hay una gran carencia de personal para cubrir todos los turnos, por lo que el $28 \%$ del personal se ve comprometido a ampliar sus horarios a un turno mixto.

\section{Capacitación para el trabajo}

De acuerdo con las Directrices IFLA/Unesco para el desarrollo del servicio de bibliotecas públicas

La capacitación es un elemento vital de las actividades de una biblioteca pública. Debe existir un programa planificado y permanente de capacitación para el personal en todos los niveles, destinado a los empleados que trabajan a tiempo completo o a tiempo parcial. La rápida evolución de la tecnología de la información acentúa aún más la necesidad de una capacitación periódica, y los programas de formación deben tener en cuenta la importancia del trabajo en red y del acceso a otras fuentes de información. ${ }^{17}$

Debido a que el bibliotecario resulta ser un agente intermediario entre los recursos de información y el usuario, es de suma relevancia que se encuentre sujeto a una formación permanente, a fin de que pueda ofrecer servicios adecuados. $^{18}$ Sin embargo, la situación de la capacitación en las bibliotecas públicas en México ofrece, según la encuesta, un panorama aún deficiente, pues la mitad del personal que labora en ellas (51\%) reporta haber recibido capacitación en pocas ocaciones (Figura 6).

\section{¿Con qué frecuencia asiste \\ a cursos de capacitación?}

Respondido: 35 Omitido 0

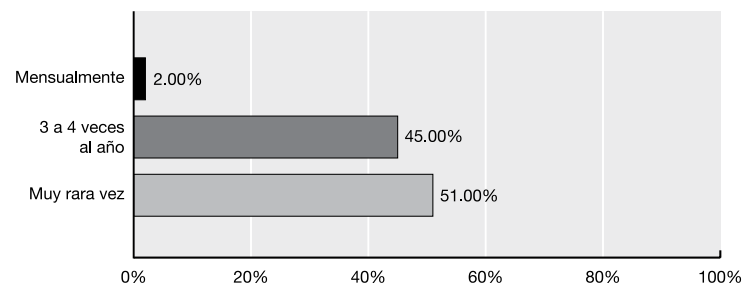

Figura 6. Gráfico de la capacitación recibida

17 Gill, op. cit., p. 59.

18 Federación Internacional de Asociaciones de Bibliotecarios y Bibliotecas, op. cit., p. 3. 
La otra mitad reporta haber recibido capacitación tres o cuatro veces al año (45\%); sin embargo, estos cursos suelen tener una duración de 3 a 5 días, con lo cual resulta mínima la formación que se pueda recibir. Los temas que se abordan suelen ser prioritariamente sobre los servicios bibliotecarios, fomento a la lectura y actividades operativas como el préstamo; por otra parte, se han establecido cursos de capacitación a lo largo de la República en torno al manejo de las tecnologías de la información, específicamente la utilización de los Módulos de Servicios Digitales.

El valor de la capacitación o formación -término más apropiado- es indispensable, ya que el personal sujeto a capacitación permanente propiciará que se oferten cada vez mejores servicios, con mayor calidad y diversidad para todos los ciudadanos.

En nuestro país son mínimos los casos en que el personal se capacita mensualmente (2\%), esta situación resulta problemática pues al no haber capacitación continua, las funciones del personal, los servicios y los procesos bibliotecarios irán en detrimento, incidiendo de manera directa en la satisfacción del usuario, quien al no encontrar respuesta a sus necesidades y expectativas comenzará a recurrir a otras instancias.

\section{Salario}

El salario que recibe el personal de las bibliotecas públicas debe estar en relación con las otras variables abordadas, por ejemplo, debe corresponder al nivel de escolaridad del individuo, así como a las competencias y habilidades que posea.

El salario es un factor determinante para el desempeño del personal, pues un salario justo y equitativo que permita cubrir las necesidades personales sin realizar otra ocupación laboral para solventar sus gastos propiciará que el bibliotecario desarrolle su labor con mayor dedicación y compromiso.

Las condiciones laborales constituyen elementos indispensables para el desempeño de las actividades bibliotecarias, por ello, todo el personal de la biblioteca pública debe trabajar en condiciones satisfactorias no sólo en cuestión de seguridad, higiene y espacios, sino también en cuestión de salarios, ya que como lo señala Phillip Gill, "los salarios deben ser apropiados en función del trabajo realizado y ser competitivos con los que corresponden a otros empleos similares en la comunidad". ${ }^{19}$ 
No obstante lo anterior, como puede observarse en la Figura 7, el salario de más de la mitad del personal que labora en las bibliotecas públicas del país es de \$2 000 a \$3 000 mensuales. En contraparte, cerca de la tercera parte de los encuestados dijeron estar por encima de la percepción de este grupo salarial.

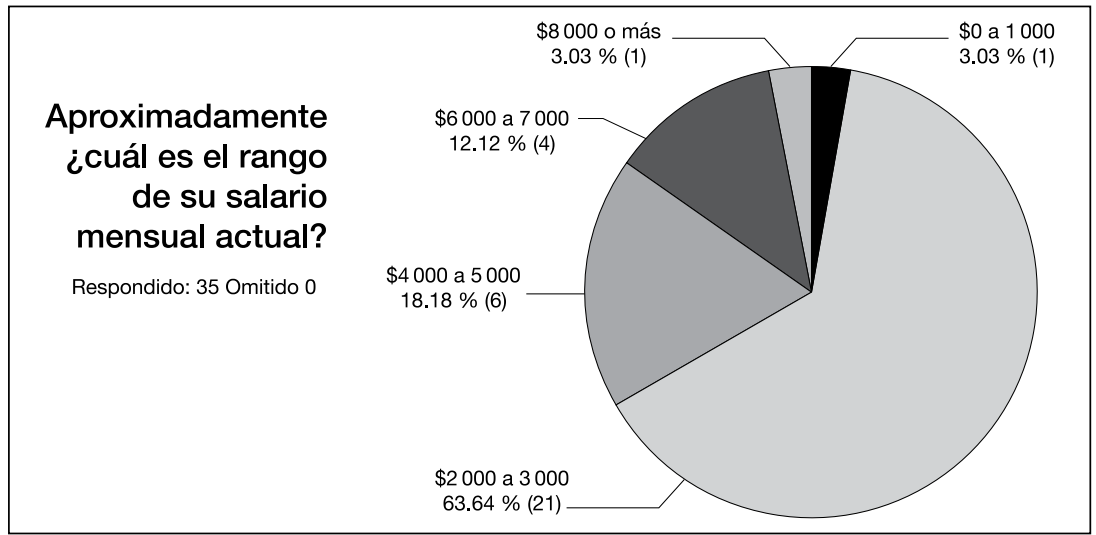

Figura 7. Salario

El estudio arrojó resultados coincidentes en relación al tipo de puesto y al salario, con esto se tiene que quienes poseen el cargo de auxiliar de biblioteca (que son la mayoría) ganan entre $\$ 2000$ y $\$ 3000$ realizando actividades como el servicio de préstamo o la atención del Módulo de Servicios Digitales; algunos de estos auxiliares bibliotecarios poseen estudios de licenciatura, mientras que otros sólo cuentan con la secundaria, a pesar de ello todos perciben el mismo salario.

En México, la Ley General de Bibliotecas (1988) no especifica bajo qué criterios habrá de ser remunerado el personal ni qué elementos serán considerados para ello; aunado a lo anterior, hasta ahora tampoco se ha desarrollado una política que incluya una tabulación y un análisis de salarios para bibliotecarios.

Debido a esta situación, la remuneración que los bibliotecarios obtienen por su trabajo es desigual y está relacionada con la región en que se encuentra la biblioteca, así como con la administración local en turno y el presupuesto asignado a la biblioteca.

Actualmente se sugiere el empleo de un sistema de compensación por competencias, el cual implica considerar factores determinantes para el buen funcionamiento de la organización tales como las habilidades, los conocimientos y las actitudes del personal, que en su conjunto constituyen compo- 
nentes esenciales para el desempeño y bajo los cuales debe establecerse una remuneración adecuada. ${ }^{20}$

En este sentido, los resultados obtenidos arrojan casos en los que una bibliotecaria de Durango, con estudios de licenciatura, trabajando en un turno mixto de lunes a viernes, gana alrededor de $\$ 3000$ mensuales y ocupa el cargo de auxiliar bibliotecario; mientras que en Campeche, una bibliotecaria encargada de una biblioteca pública municipal gana alrededor de $\$ 7000$ mensuales por trabajar durante los fines de semana, pero contando con estudios de licenciatura, diplomados y certificación como bibliotecaria; así también tenemos que otra bibliotecaria con una antigüedad de 20 a 30 años de trabajo en la biblioteca, con carrera técnica y encargada del Módulo de Servicios Digitales, gana menos de $\$ 2000$ mensuales.

Como puede observarse, hace falta establecer una valoración de puestos de trabajo, estudios comparativos y una política bibliotecaria acorde a las demandas del país, a las competencias de los bibliotecarios y a las tareas que se llevan a cabo en las bibliotecas públicas. Sin lugar a dudas, los resultados aquí plasmados invitan a realizar más investigaciones y aportaciones al respecto. En otras indagaciones se deberá conocer más sobre esta variable, así como en qué medida se correlaciona este hallazgo con la escolaridad y las funciones que realizan quienes ocupan puestos en las bibliotecas públicas.

\section{Cuarta variable. Habilidades para el trabajo}

Las bibliotecas públicas, como instituciones sociales, prestan sus servicios a una comunidad de usuarios con necesidades y características diversas. El personal que labora en las bibliotecas públicas debe poseer un conjunto de aptitudes y cualidades que lo ayuden a dar una mejor respuesta a las demandas de una comunidad cada vez más cambiante. Las Directrices IFLA/Unesco para el desarrollo del servicio de bibliotecas públicas proporcionan un listado de las principales cualidades y aptitudes requeridas para el personal de una biblioteca pública: ${ }^{21}$

- La capacidad de comunicarse positivamente con otras personas.

- La capacidad de comprender las necesidades de los usuarios.

- La capacidad de cooperar con personas y grupos en la comunidad.

- El conocimiento y la comprensión de la diversidad cultural.

- El conocimiento del material que constituye el acervo de la biblioteca y el modo de acceder a él. 
- El conocimiento de los principios del servicio público y la adhesión a ellos.

- La capacidad de trabajar con otros para prestar servicios de biblioteca eficaces.

- Aptitudes de organización con flexibilidad para definir cambios y ponerlos en práctica.

- Imaginación, visión y apertura a nuevas ideas y prácticas.

- Facilidad para modificar los métodos de trabajo a fin de responder a nuevas situaciones.

- El conocimiento de las tecnologías de la información y la comunicación.

A partir de este listado se han establecido las siguientes habilidades:

1. Cooperar y trabajar con el resto del personal.

2. Comunicarse positivamente con otras personas.

3. Atender eficazmente las necesidades de los usuarios.

4. Conocer las colecciones de la biblioteca.

5. Proponer nuevas ideas para la biblioteca.

6. Manejar las tecnologías de la información.

En la Figura 8 puede observarse que el personal se percibe de manera muy positiva en relación a sus habilidades, exceptuando las dos últimas.

\section{A continuación indique con sinceridad cómo considera su habilidad para...}

Respondido: 35 Omitido 0

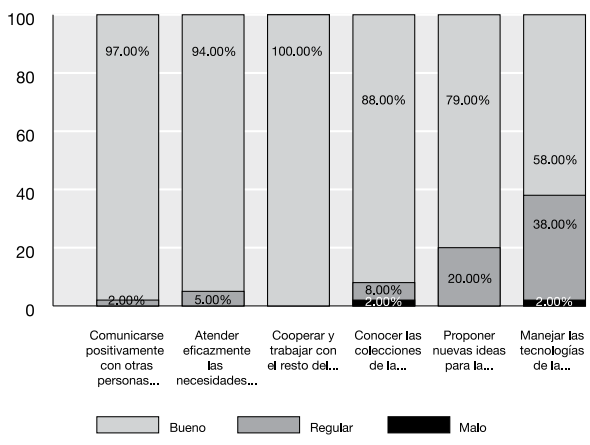

Figura 8. Gráfico de habilidades para el trabajo 
Aquellas habilidades en las que más del $90 \%$ se califica como bueno son las siguientes:

- Cooperar y trabajar con el resto del personal (bueno $=100 \%$ ).

- Comunicarse positivamente con otras personas (bueno = $97 \%$ ).

- Atender eficazmente las necesidades de los usuarios (bueno = $94 \%$ ).

- Conocer las colecciones de la biblioteca (bueno = $88 \%$ ).

No obstante, en cuanto a proponer nuevas ideas para la biblioteca, el $20 \%$ señaló tener una habilidad regular, mientras que el manejo de las tecnologías de la información fue la habilidad con la autovaloración más baja, ya que el $40 \%$ de los bibliotecarios se declaró con una habilidad regularmente mala.

El factor de las habilidades se encuentra vinculado con otras de las variables mencionadas anteriormente: la antigüedad, la escolaridad y la edad.

Al analizar los resultados puede constatarse que muchos de los bibliotecarios que consideran ser buenos en las cinco habilidades tienen una antigüedad de 10 a 20 años de trabajo en la biblioteca; asimismo, dicho personal manifiesta no recibir cursos de capacitación continua, por lo que puede deducirse que la misma experiencia en el trabajo ha contribuido a su desarrollo y a la adquisición de nuevos conocimientos que ellos mismos, por iniciativa propia y en beneficio de la realización de sus labores, han estado desarrollando.

Por otra parte, la mayoría del personal que tiene una antigüedad menor a 5 años de trabajo en la biblioteca refiere poseer pocas habilidades para conocer las colecciones de la biblioteca y proponer nuevas ideas para el desempeño de las actividades en la institución.

En lo concerniente a la edad, los resultados revelan una marcada tendencia que sostiene que a mayor edad de los bibliotecarios (específicamente aquellos mayores de 40 años) es menor la habilidad que poseen para manejar las tecnologías de la información. Aunado a ello, son pocos los cursos de capacitación que se reciben, hecho que conlleva a una permanente desactualización y rezago en este rubro.

Se reporta también que se suele asignar a los jóvenes que poseen entre 21 y 29 años como responsables de los Módulos de Servicios Digitales, y son ellos quienes en su mayoría se consideran como buenos en el dominio de las tecnologías de la información; sin embargo, ellos mismos manifiestan que tienen poca habilidad para el conocimiento de las colecciones de la biblioteca.

Los resultados obtenidos con respecto a las habilidades del personal pueden permitir un desarrollo más exacto con respecto a la capacitación de los 
bibliotecarios, pues en la medida en que se identifiquen las áreas de mejora y las competencias del personal, será posible diseñar planes de formación continua focalizados.

\section{Quinta variable. Funciones que realizan}

Para el estudio de este factor, con base en las Directrices IFLA/Unesco para el desarrollodelserviciodebibliotecaspúblicas ${ }^{22}$ sehacontempladounaseriede funciones consideradas como las más comunes en bibliotecas públicas (Tabla 2). A partir de este listado se han establecido cuatro grupos de actividades primordiales.

Tabla 2. Gráfico de funciones que realiza el personal

\begin{tabular}{|c|c|c|}
\hline \multicolumn{3}{|c|}{$\begin{array}{l}\text { De la siguiente lista de actividades, } \\
\text { marque aquellas que lleve a cabo con mayor frecuencia }\end{array}$} \\
\hline Opciones de respuesta & \multicolumn{2}{|c|}{ Respuestas } \\
\hline Intercalar los libros en la estantería & $85.71 \%$ & 30 \\
\hline Realizar el préstamo de libros a los usuarios & $77.14 \%$ & 27 \\
\hline Ayudar a los usuarios a encontrar la información que necesitan & $88.57 \%$ & 31 \\
\hline Atender la sala de cómputo de la biblioteca & $57.14 \%$ & 20 \\
\hline Efectuar la limpieza de las instalaciones de la biblioteca & $74.29 \%$ & 26 \\
\hline Velarporlaseguridaddelasinstalacionesylacomunidaddelabiblioteca & $77.14 \%$ & 27 \\
\hline Vigilar y controlar la entrada de usuarios a la biblioteca & $82.86 \%$ & 29 \\
\hline Administrarlaadquisiciónderecursosymaterialesparalabiblioteca & $74.29 \%$ & 26 \\
\hline Analizar las necesidades de información de la comunidad & $62.86 \%$ & 22 \\
\hline Formular y aplicar políticas para ampliar los servicios & $65.71 \%$ & 23 \\
\hline Planificar servicios destinados al público & $71.43 \%$ & 25 \\
\hline Manejar el presupuesto de la biblioteca & $25.71 \%$ & 9 \\
\hline Administrar las relaciones entre el personal & $42.86 \%$ & 15 \\
\hline Gestionar las relaciones con otras bibliotecas & $45.71 \%$ & 16 \\
\hline Catalogar y clasificar el material de la biblioteca & $77.14 \%$ & 27 \\
\hline Etiquetar los libros y otros documentos & $82.86 \%$ & 29 \\
\hline Encuadernar los libros de la biblioteca & $34.29 \%$ & 12 \\
\hline Hacer carteles y material de difusión para la biblioteca & $65.71 \%$ & 23 \\
\hline $\begin{array}{l}\text { Participarenactividadesdeextensiónbibliotecaria(obrasdeteatro,música, } \\
\text { talleres, cine, cuentacuentos, etc.) }\end{array}$ & $68.57 \%$ & 24 \\
\hline Total de encuestados: 35 & & \\
\hline
\end{tabular}

Al respecto de las funciones que realiza el personal en la biblioteca pública encontramos que de un total de 19 que fueron propuestas en el reactivo, más del $80 \%$ de los encuestados declara realizar cuatro actividades: 
Grupo A

- Intercalar los libros en la estantería (= $85.71 \%$ ).

- Ayudar a los usuarios a encontrar la información que necesitan (= $88.57 \%)$.

- Vigilar y controlar la entrada de usuarios a la biblioteca (= $82.86 \%)$.

- Etiquetar los libros y otros documentos (= 82.86\%).

Entre el $70 \%$ y el $79 \%$ consideran seis actividades:

Grupo B

- Realizar el préstamo de libros a los usuarios (=77.14\%).

- Catalogar y clasificar el material de la biblioteca (77.14\%).

- Efectuar la limpieza de las instalaciones de la biblioteca (=74.29\%).

- Velar por la seguridad de las instalaciones y la comunidad de la biblioteca (= 77.14\%).

- Administrar la adquisición de recursos y materiales para la biblioteca (=74.29\%)

- Planificar servicios destinados al público (=71.43\%).

Entre el $50 \%$ y el $69 \%$ indican otras cinco actividades:

Grupo C

- Participar en actividades de extensión bibliotecaria (=68.57\%).

- Hacer carteles y material de difusión para la biblioteca (=65.71\%).

- Formular y aplicar políticas para ampliar los servicios (=65.71\%).

- Analizar las necesidades de información de la comunidad (=62.86\%).

- Atender la sala de cómputo de la biblioteca (= 57.14\%).

Finalmente, cuatro actividades son mencionadas por menos del $50 \%$ :

Grupo D

- Gestionar las relaciones con otras bibliotecas (= $45.71 \%)$.

- Administrar las relaciones entre el personal (=42.86\%).

- Encuadernar los libros de la biblioteca (= 34.29\%).

- Manejar el presupuesto de la biblioteca (= $25.71 \%)$.

La mayoría del personal realiza las actividades del Grupo A, que se considerarían las básicas de cualquier biblioteca pública. En contraparte, las del Grupo B están más dedicadas para determinados procesos y servicios. Así, por ejemplo, algunas bibliotecas catalogan y clasifican sus materiales. 
Las actividades del Grupo $C$ se llevan a cabo en muchas bibliotecas públicas y se considera que cada día están tomando mayor relevancia.

Las actividades del Grupo D son de carácter directivo o especializado (encuadernación). Sin embargo, es de llamar la atención que algunas del Grupo B también pueden considerarse directivas, tales como administrar adquisiciones y planificar servicios.

\section{Conclusiones}

El presente estudio muestra parcialmente la situación de los recursos humanos en las bibliotecas públicas de México y ha permitido detectar la existencia de un perfil laboral sobre el que se requiere investigar con mayor amplitud y profundidad. De esta manera, un ejemplo del perfil del personal de biblioteca pública que resulta de este estudio es el siguiente:

Una bibliotecaria adulta con escolaridad media, con poca antigüedad trabajando en la institución, con un horario variable, aunque preferiría el matutino; que no habría recibido capacitación para este trabajo, ganando menos de dos salarios mínimos, con cuatro habilidades para el trabajo y falta de desarrollo en el manejo de las tecnologías de la información. Realiza tareas del Grupo A.

Lo que aquí se reporta es exploratorio y no es conclusivo. De lo que hemos indicado a lo largo de este informe los siguientes asuntos requieren más investigación:

- Cómo es la capacitación o formación continua del personal, pues la mitad reporta no haberla recibido.

- Cuál es la situación salarial del personal que percibe menos, qué prestaciones tiene y en qué medida vive con su sueldo.

- Si existe alguna correlación entre el salario y la escolaridad del personal de las bibliotecas públicas.

- En qué medida se pueden reforzar las dos habilidades del personal con los puntajes más bajos: proposición de nuevas ideas para la biblioteca y manejo de tecnologías de la información.

- Cómo realiza el personal las actividades en las bibliotecas públicas. En particular, interesa conocer las competencias que tienen para ello.

- Cómo hacer efectivas las propuestas para la mejora de los recursos humanos de las bibliotecas públicas. 
Así, luego de haber conocido los resultados de este primer abordaje exploratorio sobre la situación que existe en el ámbito de los recursos humanos en las bibliotecas públicas mexicanas, consideramos necesario proponer las acciones siguientes para su mejora:

- Establecer una base de datos del personal que labora en las bibliotecas públicas de México, en la que consten los factores que aquí indicamos, así como otros relacionados con la administración y desarrollo de los recursos humanos.

- Establecer fórmulas para la selección de personal, la evaluación del rendimiento y la valuación de puestos de trabajo que conecten con otros factores.

- Establecer una metodología para el análisis y descripción de los perfiles de los recursos humanos de las bibliotecas públicas, con la finalidad de asegurar la calidad en el desempeño y proyectar la mejora continua de los servicios.

- Establecer escalas de retribución para el personal de las bibliotecas públicas en referencia a los salarios mínimos por zona y otros factores como la escolaridad o las competencias.

- Desarrollar herramientas de enseñanza-aprendizaje innovadoras para la formación continua y el desarrollo de las competencias de los recursos humanos en las bibliotecas públicas.

- Desarrollar y aplicar periódicamente encuestas sobre clima laboral.

- Incorporar elementos de planeación estratégica para el desarrollo de los recursos humanos.

En los intentos para emprender estas mejoras, no debe olvidarse que

las bibliotecas, como entidades de cambio, tienen la obligación de evaluar su propio desempeño y su impacto en la sociedad. Por ello, es necesario y fundamental adaptar e integrar paradigmas de gestión y desarrollo de personal que se hayan probado con éxito en otras organizaciones o instituciones a nivel mundial. ${ }^{23}$

\section{Referencias bibliográficas}

Chiavenato, Idalberto, Administración de recursos humanos, 5a. ed., Colombia: McGraw-Hill, 2000. 
Chiavenato, Idalberto, Gestión del talento humano, 3a. ed., México: McGraw Hill, 2009.

Dessler, Gary; Varela Juárez, Ricardo A., Administración de recursos humanos: enfoque latinoamericano, 2a. ed., México: Pearson Educación, 2004.

DirectricesIFLA/Unesco paraeldesarrollodelserviciodebibliotecaspúblicas, IFLA/Unesco, 2001. Disponible en: http://unesdoc.unesco. org/images/0012/001246/124654s.pdf [Fecha de consulta: 26 de marzo de 2014].

Federación Internacional de Asociaciones de Bibliotecarios y Bibliotecas, Manifiesto de la IFLA/Unesco sobre la biblioteca pública, 1994, trad. de Fundación Germán Sánchez Ruipérez, 2004. Disponible en: http://www.ifla.org/ES/node/7271 [Fecha de consulta: 20 de octubre de 2013].

Gill, P., Directrices IFLA/Unesco para el desarrollo del servicio de bibliotecas públicas, 2a. ed., México: Consejo Nacional para Cultura y las Artes, Dirección General de Bibliotecas; La Haya: IFLA/Unesco, 2007.

González Amador, Roberto, “En México 4 de cada diez personas no tienen para comprar alimentos", en La Jornada, miércoles 19 de marzo de 2014. Disponible en: http://www.jornada.unam.mx/20 14/03/19/economia/026n2eco [Fecha de consulta: 26 de marzo de 2014].

Hernández Pacheco, F., El bibliotecólogo emprendedor: un reto del siglo XXI, México: Colegio Nacional de Bibliotecarios, Universidad Latinoamericana, 2000.

- Gestióny desarrollo de recursos humanos en bibliotecas, México: Secretaría de Educación Pública, ENBA, Universidad Complutense de Madrid, Library Outsourcing, 2012.

Hernández Silva, F. E. y Martí Lahera, Y., "Conocimiento organizacional: la gestión de los recursos y el capital humano", en Acimed, 14 (1), 2006. Disponible en: http://bvs.sld.cu/revistas/aci/vol14_1_06 /aci03106.htm [Fecha de consulta: 20 de octubre de 2013].

Los RecursosHumanos.com, ¿A qué se denomina recursos humanos?, 2008. Disponible en: http://www.losrecursoshumanos.com/con tenidos/5403-a-que-se-denomina-recursos-humanos.html[Fecha de consulta: 20 de octubre de 2013].

Matus Sepúlveda, G., "Perspectivas en la formación de recursos humanos en bibliotecología", en Biblios, 14, 2002. Disponible en: http:// eprints.rclis.org/5472/1/B14-04.pdf [Fecha de consulta: 20 de octubre de 2013].

México, Ley General de Bibliotecas, 2009. Disponible en: http://www. diputados.gob.mx/LeyesBiblio/pdf/134.pdf [Fecha de consulta: 20 de octubre de 2013].

México, Ley de Bibliotecas del Distrito Federal, 2009. Disponible en: http://www.consejeria.df.gob.mx/images/leyes/leyes/LEYDEBI BLIOTECASDELDISTRITOFEDERAL.pdf [Fecha de consulta: 20 de octubre de 2013]. 
Encuesta aplicada a los recursos humanos en la RNBP

Los recursos humanos en las bibliotecas públicas de México

1. ¿En qué biblioteca trabaja y qué puesto desempeña?

Bibliotecapúblicaenlaquetrabaja

Cargoopuestodetrabajo

MunicipiooDelegación

Estado o Región

2. ¿Cuál es su edad?

* 17 o menos

. $18-20$

. $21-29$

. $30-39$

. $40-49$

. 50-59

. 60 o más

3. Genero

. Hombre

. Mujer

4. Escolaridad / último grado de estudios:

* Sin estudios

. Primaria

. Secundaria

Preparatoria

Maestría

"Carrera técnica "Doctorado

Licenciatura

Enelcasodecarreratécnica,licenciaturaoposgrado,especifiqueenquéáreaocarrera:

5. ¿Cuál es su horario laboral?

Seleccione el horario en el que

Turno

Días laborales

trabaja en la biblioteca

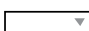

\section{$\nabla$}

6. Aproximadamente, ¿cuál es el rango de su salario mensual actual?

7. ¿Cuánto tiempo lleva trabajando en la biblioteca?

- 5 años o menos

. 6 a 10 años 
. 10 a 20 años

. 20 a 30 años

* Más de 30 años

8. ¿Con qué frecuencia asiste a cursos de capacitación?

.. Mensualmente .3 a 4 veces al año . Muy rara vez

9. A continuación indique con sinceridad cómo considera su habilidad para

- Comunicarse positivamente con otras personas Bueno Regular Malo

- Atender eficazmente las necesidades de los usuarios

- Cooperar y trabajar con el resto del personal

- Conocer las colecciones de la biblioteca

- Proponer nuevas ideas para la biblioteca

- Manejar las tecnologías de la información

10. De la siguiente lista de actividades, marque aquellas que lleve a cabo con mayor frecuencia

- Intercalar los libros en la estantería

- Realizar el préstamo de libros a los usuarios

- Ayudar a los usuarios a encontrar la información que necesitan

- Atender la sala de cómputo de la biblioteca

- Efectuar la limpieza de las instalaciones de la biblioteca

* Velar por la seguridad de las instalaciones y la comunidad de la biblioteca

- Vigilar y controlar la entrada de usuarios a la biblioteca

- Administrar la adquisición de recursos y materiales para la biblioteca

- Analizar las necesidades de información de la comunidad

- Formular y aplicar políticas para ampliar los servicios

- Planificar servicios destinados al público

- Manejar el presupuesto de la biblioteca

- Administrar las relaciones entre el personal

- Gestionar las relaciones con otras bibliotecas

- Catalogar y clasificar el material de la biblioteca

- Etiquetar los libros y otros documentos

- Encuadernar los libros de la biblioteca

- Hacer carteles y material de difusión para la biblioteca

.- Participarenactividadesdeextensiónbibliotecaria(obrasdeteatro,música,talleres, cine, cuentacuentos, etc.)

Otro (especifique) 
Para citar este artículo como revista electrónica:

Hernández Pacheco, Federico y Diana Quezada Escamilla. 2016.

"Los recursos humanos en las bibliotecas públicas de México: una investigación exploratoria".Investigación Bibliotecológica:Archivonomía,Bibliotecologíaelnformación.68:17-50.Aquíseagrega la dirección electrónica (Consultado el día-mes-año)

Para citar este artículo tomado de un servicio de información:

Hernández Pacheco, Federico y Diana Quezada Escamilla. 2016. "Los recursos humanos en las bibliotecas públicas de México: una investigación exploratoria".Investigación Bibliotecológica:Archivonomía, Bibliotecología e Información. 68: 17-50. En: Aquí se agregaelnombredelserviciodeinformaciónyladirecciónelectrónica (Consultado el día-mes-año) 